\title{
Identification of Epitopes In The Nucleocapsid Protein of Nipah Virus Using a Linear Phage-Displayed Random Peptide Library
}

\begin{abstract}
A random peptide library of heptamers displayed on he surface of M13 bacteriophage was used to identify specific epitopes of antibodies in pooled sera of swine naturally infected by Nipah virus. The selected heptapeptides were aligned with proteinsequences of Nipah virus and several putative epitopes were indentified within the nucleocapsid protein. A total of 41 to $60(68 \%)$ selected phage clones had inserts resembling a region with the sequence SNRTQGE, located at the C-terminal end (amino acids 503-509) of the nucleocapsid protein. The binding of antibodies in the swine and human antisera to the phage clone was inhibited by synthetic peptide corresponding to this region. Epitopes identified by phage display are consistent with the predicted antigenic sites for the Nipah virus nucleocapsid protein. The selected phage clone used as a coating antigen discriminated swine and human Nipah virus sera-positive from sera-negative samples exhibit characteristics, which might be attractive for diagnostic tests.
\end{abstract}

